\title{
A Rapid Association Test Procedure Robust under Different Genetic Models Accounting for Population Stratification
}

\author{
Wenan Chen ${ }^{a}$ Xiangning Chen ${ }^{b}$ Kellie J. Archer ${ }^{a}$ Nianjun Liu ${ }^{c}$ Qizhai Li ${ }^{d}$ \\ Zhongming Zhao ${ }^{\text {e, }}$ Shumei Sun ${ }^{a}$ Guimin Gao ${ }^{a}$ \\ Departments of a Biostatistics, and b Psychiatry and Human Molecular Genetics, School of Medicine, Virginia \\ Commonwealth University, Richmond, Va., and 'Department of Biostatistics, University of Alabama at Birmingham, \\ Birmingham, Ala., USA; ${ }^{d}$ Academy of Mathematics and Systems Science, Chinese Academy of Sciences, Beijing, \\ PR China; ' Departments of Biomedical Informatics, Cancer Biology, and Psychiatry, School of Medicine, and \\ fCenter for Quantitative Sciences, Vanderbilt University, Nashville, Tenn., USA
}

\section{Key Words}

Generalized sequential Bonferroni procedure .

Genome-wide association studies · Hardy-Weinberg

disequilibrium trend test - Robust test $\cdot$ Recessive model

\begin{abstract}
Objective: For genome-wide association studies (GWAS) using case-control data with stratification, a commonly used association test is the generalized Armitage (GA) trend test implemented in the software EIGENSTRAT. The GA trend test uses principal component analysis to correct for population stratification. It usually assumes an additive disease model and can have high power when the underlying disease model is additive or multiplicative, but may have relatively low power when the underlying disease model is recessive or dominant. The purpose of this paper is to provide a test procedure for GWAS with increased power over the GA trend test under the recessive and dominant models, while maintaining the power of the GA trend test under the additive and multiplicative models. Methods: We extend a HardyWeinberg disequilibrium (HWD) trend test for a homogeneous population to account for population stratification,
\end{abstract}

and then propose a robust association test procedure for GWAS that incorporates information from the extended HWD trend test into the GA trend test. Results and Conclusions: Our simulation studies and application of our method to a GWAS data set indicate that our proposed method can achieve the purpose described above.

Copyright $\odot 2013$ S. Karger AG, Basel

\section{Introduction}

In genome-wide association studies (GWAS) with case-control designs, population stratification can happen when the participants are sampled from different subpopulations with significant allele frequency differences [1]. Population stratification can be a confounding factor in the association test and may produce spurious associations if not properly corrected [2-4]. Many methods have been developed to correct for stratification in association studies $[3,5,6]$. One of the most widely used methods is the generalized Armitage (GA) trend test implemented in the software EIGENSTRAT that uses principal component analysis to correct for

\section{KARGER}

E-Mail karger@karger.com

www.karger.com/hhe
(C) 2013 S. Karger AG, Basel

0001-5652/13/0751-0023\$38.00/0
Guimin Gao

Department of Biostatistics, School of Medicine

Virginia Commonwealth University, PO Box 980032

Richmond, VA 23298-0032 (USA)

E-Mailggao3@vcu.edu 
population stratification [3]. The GA trend test usually assumes that the underlying disease model is additive [3]. In this paper, we use 'GA trend test' to denote the GA trend test assuming an additive model. One of the advantages of the GA trend test is that when the underlying disease model is additive or multiplicative, it can have high power in detecting causal variants. However, when the underlying disease model is dominant or recessive, it can have low power.

Some association tests that are robust to different genetic models have been developed for homogenous populations [7-10]. For example, the MAX3 test statistic [7, 8] uses the maximum of the three Armitage trend test statistics [11] that assume the additive, dominant and recessive model, respectively. MAX3 has been extended to use top principal components (PCs) of genome-wide genotypes as covariates to correct for stratification [12]. This extended MAX3 method is referred to as 'MAX3PC' in this paper. However, MAX3-PC has 2 limitations: (1) compared to the GA trend test, when the underlying disease model is additive or multiplicative, MAX3-PC can have relatively lower power; and (2) it can be computationally intensive for GWAS, especially for data with more than 1,000,000 SNPs. Since the additive/multiplicative genetic model is often assumed and fits well for most identified genetic variants [13], in this article, we intend to propose a computationally efficient method for GWAS that can maintain the power of the GA trend test under the additive and multiplicative models, while it has improved power over the GA trend test under the recessive and dominant models.

To achieve this goal, first, we extend the Hardy-Weinberg disequilibrium (HWD) trend test by Song and Elston [14] for homogenous populations to account for population stratification. The 'extended HWD trend test' can provide useful information under the dominant or recessive models which is different from that provided by the GA trend test. Second, for GWAS we apply the generalized sequential Bonferroni (GSB) procedure to incorporate information from the extended HWD trend test into the GA trend test. Simulation studies indicate that the proposed GSB procedure for GWAS can control the family-wise error rate (FWER) when stratification exists; it has comparable power to that of the GA trend test, when the underlying model is additive or multiplicative, and has higher power than the GA trend test when the underlying model is dominant or recessive. Simulation studies also indicate that MAX3-PC by So and Sham [12] has relatively lower power than the GA trend test and the proposed GSB procedure under the additive and multiplica- tive models. Finally, we applied our proposed method to 4 data sets provided by the Wellcome Trust Case-Control Consortium (WTCCC) [13].

\section{Methods}

We first extend the HWD trend test by Song and Elston [14] to account for population stratification, and then propose a GSB procedure for GWAS that incorporates information from the extended HWD trend test into the GA trend test. In this study, we assume independence among markers and ignore the LD among markers in the GWAS data.

\section{Extended HWD Trend Test}

Assuming that cases and controls are sampled from the same general homogenous population, Song and Elston [14] proposed a HWD trend test comparing the HWD coefficient in cases, $D_{\text {case }}$, with the HWD coefficient in controls, $D_{\text {control }}$. For a SNP with allele $A$ and $B$, we denote the genotype frequencies of $A A, A B, B B$ in cases by $p_{A A}, p_{A B}, p_{B B}$ and in controls by $q_{A A}, q_{A B}, q_{B B}$. Then we have $D_{\text {case }}=p_{B B}-\left(p_{A B} / 2+p_{B B}\right)^{2}$ and $D_{\text {control }}=q_{B B}-\left(q_{A B} / 2+q_{B B}\right)^{2}$. Let $\Delta$ be $D_{\text {case }}-D_{\text {control }}$. The null hypothesis $\left(H_{0}\right)$ is: $\Delta=0$, which corresponds to testing whether the HWD coefficient for the test marker in the controls is equivalent to that in cases; if not, the test marker may be associated with the disease. The HWD trend test statistic is given by:

$$
T_{\text {HWDTT }}=\hat{\Delta} / \sqrt{\operatorname{Var}_{H_{0}}\left(\hat{\Delta}_{i}\right)},
$$

where $\hat{\Delta}=\hat{D}_{\text {case }}-\hat{D}_{\text {control }}$, and $\hat{D}_{\text {case }}$ and $\hat{D}_{\text {control }}$ are the maximum likelihood estimates of $D_{\text {case }}$ and $D_{\text {control }}$, respectively. $V a \hat{r}_{H_{0}}\left(\hat{\Delta}_{i}\right)$ is the estimate of the variance of $\hat{\Delta}$ under $H_{0}$ [for details, see 14]. $T_{\text {HWDTT }}$ asymptotically follows a standard normal distribution, and the square of the test follows the $\chi^{2}$ distribution with 1 degree of freedom.

The HWD trend test statistic, $T_{\text {HWDTT }}$, can be used to test the association between a marker and a disease under the assumption that the Hardy-Weinberg equilibrium holds in the general population from which the cases and controls are sampled. Song and Elston [14] stated that the HWD trend test can automatically control for the genotyping errors, if the cases and controls are genotyped by the same methods at the same time, with the cases and controls randomized. However, a weakness of the HWD trend test is that it can have an inflated type I error rate when population stratification exists (see our simulation results below). Below we extend the HWD trend test to account for population stratification.

First, we assign the cases and controls in the data to $K$ groups (or clusters) that correspond to $K$ putative subpopulations. Specifically, we calculate the top PCs, e.g. the top 10 PCs, of the genome-wide genotypes and then apply a clustering algorithm, e.g. the $k$-means clustering algorithm [15], to the top PCs to partition the individuals into $K$ groups.

In order to determine the number of subpopulations $(K)$, we use the fpc package (http://cran.r-project.org/web/packages/fpc/ index.html) that implements the $k$-means clustering algorithm with options to determine the optimal number $(K)$ of clusters [16]. More specifically, the fpc package uses the Duda-Hart test [17] to 
test if all individuals in the data are from one cluster. If they are not from one cluster, then the $k$-means clustering algorithm is executed with the cluster numbers $k=2,3, \ldots, K_{\max }$ (we set $K_{\max }=4$ in our data analysis). The Calinski-Harabasz criterion is used to determine the optimal number of clusters from 2 to $K_{\max }$ [18]. The Calinski-Harabasz criterion can be calculated as:

$$
C H(k)=\frac{S S B /(k-1)}{S S W /(N-k)},
$$

where $S S W$ is the sum of variances within the clusters, SSB is the sum of variances among the clusters, and $N$ is the number of individuals in the case-control data. The number of subpopulations is selected as:

$$
K=\arg \max C H(k) \text {. }
$$

For the cases and controls assigned to the $i$-th group where $i=$ $1,2, \ldots, K$, we define an effect size to be the difference $\left(\Delta_{i}\right)$ of the HWD coefficients between cases and controls: $\Delta_{i}=D_{\text {case }}^{i}-D_{\text {control }}^{i}$, and we denote its maximum likelihood estimation by $\beta_{i}=\hat{\Delta}_{i}$. Under $H_{0}$, the Hardy-Weinberg equilibrium holds in the $i$-th subpopulation (corresponding to the $i$-th group) and the test marker is not associated with the disease, the variance of the estimated effect, $\operatorname{Var}_{H_{0}}\left(\hat{\Delta}_{i}\right)$, can be estimated (see Song and Elston [14]). If any assigned group only contains cases, then we set the HWD coefficient for the controls to $D_{\text {control }}^{i}=0$ and use the variance $\operatorname{Var}_{H_{0}}$ $\left(\hat{D}_{\text {case }}^{i}\right)$, as given in Song and Elston [14]. If any assigned groups only contain controls, this group is removed from the analysis because subpopulations consisting of controls only do not provide information for the association study.

Following the method in Mägi and Morris's meta-analysis using a fixed effect model [19], the combined effect over the $K$ groups (or subpopulations) is given by

$$
E=\frac{\sum_{i=1}^{K} \beta_{i} w_{i}}{\sum_{i=1}^{K} w_{i}},
$$

where $w_{i}=\left[\operatorname{Va} \hat{r}_{H_{0}}\left(\hat{\Delta}_{i}\right)\right]^{-1}$ is the inverse of the estimated variance of the estimated effect for the $i$-th subpopulation. The variance of the combined effect is given by

$$
V=\left[\sum_{i=1}^{K} w_{i}\right]^{-1} .
$$

Under $H_{0}$ that the Hardy-Weinberg equilibrium holds in each of the subpopulation and the test marker is not associated with a disease, the extended HWD trend test statistic is then defined as $E^{2} / V$, which has an approximate $\chi^{2}$ distribution with 1 degree of freedom.

\section{GA Trend Test}

To effectively control false positive rates caused by population stratification, Price et al. [3] proposed the GA trend test, which is implemented in the software EIGENSTRAT. The GA trend test calculates the top PCs from genome-wide genotype values for each individual. To test the association between a marker and the disease status, the GA trend test adjusts the individual's genotype value (assuming an additive model) at the marker by regressing out the top $L$ PCs (e.g. $L=10$ ) in a linear model (see Price et al. [3]) and then adjusts the phenotypic value ( 0 for a control and 1 for a case) of the same $L$ PCs in a similar way. The GA trend test statistic is defined as $(N-L-1) R^{2}$, where $R^{2}$ is the correlation between the ancestry-adjusted genotype and ancestry-adjusted phenotype, and $N$ is the number of individuals in the case-control study. Under $H_{0}$ that the test marker is not associated with the disease, the GA trend test statistic asymptotically follows the $\chi^{2}$ distribution with 1 degree of freedom.

\section{GSB Procedure for Multiple Testing}

For testing multiple hypotheses, $H_{1}, H_{2}, \ldots, H_{m}$, Holm [20] proposed a GSB procedure by assigning different weights to hypotheses of different importance. Let $\alpha$ denote the nominal FWER, which is defined as the probability of rejecting at least 1 true null hypothesis. We describe the GSB procedure below.

Let $p_{1}, p_{2}, \ldots, p_{m}$ denote the $\mathrm{p}$ values from $m$ tests corresponding to hypotheses $H_{1}, H_{2}, \ldots, H_{m}$. Given weights, $w_{1}, w_{2}, \ldots, w_{m}$, for these tests, we define the $B$ values as $B_{i}=p_{i} / w_{i}$. First, we order the $B$ values as $B_{(1)} \leq B_{(2)} \leq \ldots \leq B_{(\mathrm{m})}$, so that $w_{(1)}, w_{(2)}, \ldots, w_{(\mathrm{m})}$ and $H_{(1)}$, $H_{(2)}, \ldots, H_{(\mathrm{m})}$ are the corresponding weights and hypotheses of the ordered $B$ values. Starting from $i=1$, given $H_{(1)}, H_{(2)}, \ldots, H_{(\mathrm{i}-1)}$ have been tested and rejected, if

$$
B_{(i)} \leq \alpha / \sum_{k=i}^{m} w_{(k)},
$$

reject $H_{(\mathrm{i})}$; otherwise, accept $H_{(\mathrm{i})}, H_{(\mathrm{i}+1)}, \ldots, H_{(\mathrm{m})}$, and stop the GSB procedure. Holm [20] proved that the GSB procedure controls the FWER at the nominal significance level $\alpha$, if the condition that the weights are independent of the $m$ tests is satisfied. From the proof by Holm [20], we can see that the GSB procedure is relatively conservative and that the condition that the weights are independent of the $m$ tests is a sufficient condition but not a necessary condition. We will show by simulation studies (see Appendix) that in some situations, even though the weights are weakly correlated with the $m$ tests, the GSB procedure can still control the FWER well. In addition, from the rejection criterion above, in order to reject hypothesis $H_{(\mathrm{i})}$, the corresponding $\mathrm{p}$ value $p_{(\mathrm{i})}$ must be less than or equal to $\alpha$, otherwise the GSB procedure will not reject hypothesis $H_{(\mathrm{i})}$.

We note that when the GSB procedure by Holm is applied to test the association at genome-wide markers, it cannot provide a $\mathrm{p}$ value for each marker, which may be needed when comparing the GSB procedure with other methods in real data analysis. Therefore, we define the adjusted $p$ value for each test in the GSB procedure, following the idea of Westfall and Young [21, p. 64]: let $\tilde{p}_{(\mathrm{i})}$ be the adjusted $\mathrm{p}$ value corresponding to $B_{(\mathrm{i})}$ and $H_{(\mathrm{i})}, i=1,2, \ldots$, $m$. Then we define

$$
\begin{aligned}
& \tilde{p}_{(1)}=\left(\sum_{i=1}^{m} w_{(i)}\right) B_{(1)}, \\
& \tilde{p}_{(2)}=\max \left(\left(\sum_{i=2}^{m} w_{(i)}\right) B_{(2)}, \tilde{p}_{(1)}\right), \ldots, \\
& \tilde{p}_{(j)}=\max \left(\left(\sum_{i=j}^{m} w_{(i)}\right) B_{(j)}, \tilde{p}_{(j-1)}\right), \ldots, \\
& \tilde{p}_{(m)}=\max \left(w_{(m)} B_{(m)}, \tilde{p}_{(m-1)}\right) .
\end{aligned}
$$


Any adjusted $\mathrm{p}$ value greater than 1.0 should be set to 1.0. The adjusted $\mathrm{p}$ values should be compared to the nominal level $\alpha$ for the entire test procedure: if an adjusted $\mathrm{p}$ value is less than or equal to $\alpha$, then reject the corresponding hypothesis.

\section{GSB Procedure for GWAS Using Case-Control Data with}

Population Stratification

Here we describe how to apply the GSB procedure for multiple testing to GWAS data with population stratification. In the original GSB procedure, Holm [20] did not describe how to estimate the weights, although the GSB procedure has the potential to improve the power of multiple hypotheses testing when prior information is available to estimate the weights. Since the extended HWD trend test may provide different information from that provided by the GA trend test, we propose to use the extended HWD trend test to calculate a weight for each marker, and use the GA trend test to calculate a $\mathrm{p}$ value for the marker. Then we use the weight to adjust the GA trend test's $p$ value in the GSB procedure. Specifically, let $q_{i}$ denote the $\mathrm{p}$ value for the $i$-th marker calculated by the extended HWD trend test. We let the weight $w_{i}$ for the $i$-th marker be the inverse of the extended HWD trend test p value $q_{i}$, i.e. $w_{i}=1 / q_{i}$. We then apply the GSB procedure to incorporate the weight into the corresponding GA trend test in GWAS.

As described in GSB Procedure for Multiple Testing, to reject $H_{0}$ that a marker is not associated with the disease, the $\mathrm{p}$ value from the GA trend test at that marker must be less than or equal to the nominal FWER $\alpha$. This property can prevent that a null marker, which usually has a $\mathrm{p}$ value from the GA trend test greater than $\alpha$ (such as 0.05), is called significant because of the extremely high weight that is calculated from the extended HWD trend test due to genotyping errors.

The extended HWD trend test can have high power under both the recessive and dominant models, but it has no (or almost no) power under the multiplicative (or additive) model. Therefore, the weights calculated from the extended HWD trend test only generate noise when the underlying disease model is multiplicative or additive. This results in that under these 2 models, the GSB procedure (using $\mathrm{p}$ values from the GA trend test) has relatively lower power than the GA trend test (with Bonferroni correction) in GWAS. On the other hand, when the underlying disease models are recessive or dominant, weights calculated from the extended HWD trend test can provide useful information, and the GSB procedure can have much higher power than the GA trend test (see our simulation results below).

Since the additive model is often assumed in association studies in the literature when the true underlying genetic model is unknown, we hope to modify the GSB procedure for GWAS so that it can maintain the power of the GA trend test under the additive and multiplicative models, but still has increased power under the dominant and recessive models. To achieve this goal, we employ a smoothing method by Roeder et al. [22] to calculate smoothed weights. Suppose $w_{i}$ is the original weight for the $i$-th marker, $\bar{w}$ is the average value of the weights for all markers. We calculate a smoothed weight for the $i$-th marker as $w_{i}^{\prime}=(1-\lambda) w_{i}+\lambda \bar{w}$, where $\lambda$ is a parameter and $0 \leq \lambda \leq 1$. We call the GSB procedure using the smoothed weights 'smooth-GSB procedure'. When $\lambda$ is close to 1 , then the smoothed weight is close to the constant $\bar{w}$, and the GSB procedure has similar results as the GA trend test. On the other hand, when $\lambda$ is close to 0 , the smoothed weight is close to the original weight $w_{i}$, and the smooth-GSB procedure has similar results as the GSB procedure using the original weight $w_{i}$. Since we hope that the smooth-GSB procedure has power close to that of the GA trend test in GWAS under the additive and multiplicative models, the value of $\lambda$ should be close to 1 . On the other hand, if $\lambda$ is very close to 1 , only a little information from the original weight (i.e. from the extended HWD trend test) can be used, this will result in that the smooth-GSB procedure does not have much increased power compared to the GA trend test under the recessive and dominant models. As a tradeoff, we would suggest the value of $\lambda$ should be greater than 0.5 but less than 0.9 .

In the GSB procedure for GWAS, if the weight calculated from the extended HWD trend test is (asymptotically) independent of the GA trend test at each marker, then the GSB procedure for GWAS can control the FWER. Joo et al. [23] proved that the HWD trend test statistic and the Cochran Armitage trend test statistic assuming the additive model are asymptotically independent in homogeneous populations. We expect that, as the extension of these 2 test statistics correcting for stratification, the extended HWD trend test statistic and the GA trend test statistic are approximately independent or at most are very weakly correlated. However, it is difficult to prove this independence theoretically. Below we will show by simulation studies that, at a marker, the correlation between the extended HWD trend test statistic and the GA trend test statistic is negligible. We will also show that in simulated case-control GWAS data, the GSB and smooth-GSB procedures can control the FWER.

\section{Simulation Studies}

\section{Simulating Case-Control Data with Stratification}

To evaluate the false positive rates and power of the extended HWD trend test and the proposed smooth-GSB procedure in case-control data with population stratification, we simulated data sets in a similar way to that in Price et al. [3]. Each data set was simulated to include 2,000 cases and 2,000 controls with 10,000 random SNPs. To simulate stratification, each individual was sampled from 1 of 2 populations. In the case group, $40 \%$ were sampled from population 1 and $60 \%$ were sampled from population 2. In the control group, $20 \%$ were sampled from population 1 and $80 \%$ were sampled from population 2 . Allele frequencies for population 1 and population 2 at each of the 10,000 random SNPs were generated using the Balding-Nichols model with $F_{S T}=0.01$. Specifically, at each random SNP, the allele frequencies for populations 1 and 2 were each drawn from a beta distribution with parameters $p\left(1-F_{S T}\right) / F_{S T}$ and $(1-p)\left(1-F_{S T}\right) / F_{S T}$, where the ancestral population allele frequency $p$ was sampled from the uniform distribution in the interval $[0.1,0.9]$. Usually the allele frequency differences between populations 1 and 2 are less than 0.1 [3]. In addition to the 10,000 random SNPs, we simulated 10 SNPs with differential frequencies in populations 1 and 2 . For each of these 10 
SNPs, the allele frequency difference between populations 1 and 2 was greater than 0.1 , to approximate the effect of selection. In our simulation, we set the allele frequency to 0.2 in population 1 and 0.8 in population 2 as a demonstration of strong selection. As an example, figure 1 shows the allele frequency differences at the 10,000 random SNPs between population 1 and population 2 in one replicate of our simulated data set. We can see that most of the allele frequency differences between the 2 populations are less than 0.1 .

\section{Type I Error Evaluation of the Original HWD Trend}

Test and the Extended HWD Trend Test

To compare the type I errors of the original HWD trend test by Song and Elston [14] and the extended HWD trend test, we chose a null SNP, i.e. a non-disease-causing SNP, from the simulated 10,000 plus 10 SNPs and evaluated the type I errors at this SNP. The null SNP was chosen in 2 ways: (1) from the 10,000 random SNPs and (2) from the 10 SNPs with differential frequencies, where we set the allele frequency as 0.2 in subpopulation 1 and 0.8 in subpopulation 2 . We simulated 10,000 replicated data sets to estimate the type I error, the results of which are showed in table 1 . We can see that with the random null SNP, both the original HWD trend test and the extended HWD trend test could control the type I error well. However, at the SNP with highly differentiated allele frequencies in the 2 subpopulations, the original HWD trend test had an obviously inflated type I error rate and the extended HWD trend test could still control the type I error.

\section{Evaluation of Correlation between the GA Trend Test} and the Extended HWD Trend Test

We estimated the correlation coefficients between the GA trend test and the extended HWD trend test under $H_{0}$ that no markers are associated with the disease and that the Hardy-Weinberg equilibrium holds in each subpopulation. Under this $H_{0}$, we generated 10,000 replicates of the case-control data with 10,000 plus 10 SNPs (see Simulating Case-Control Data with Stratification above). For each SNP, we calculated the GA trend test statistic and the extended HWD trend test statistic. The sample Spearman's rank correlation coefficient between the GA trend test and the extended HWD trend test was calculated for each SNP based on the 10,000 replicates. The mean and variance of the absolute values of Spearman's rank correlation coefficients for the 10,000 plus 10 markers were 0.00799 and 0.0060 , respectively. These values indicate that the correlation between the 2 statistics is negligible.

Robust Association Test Procedure

Accounting for Stratification

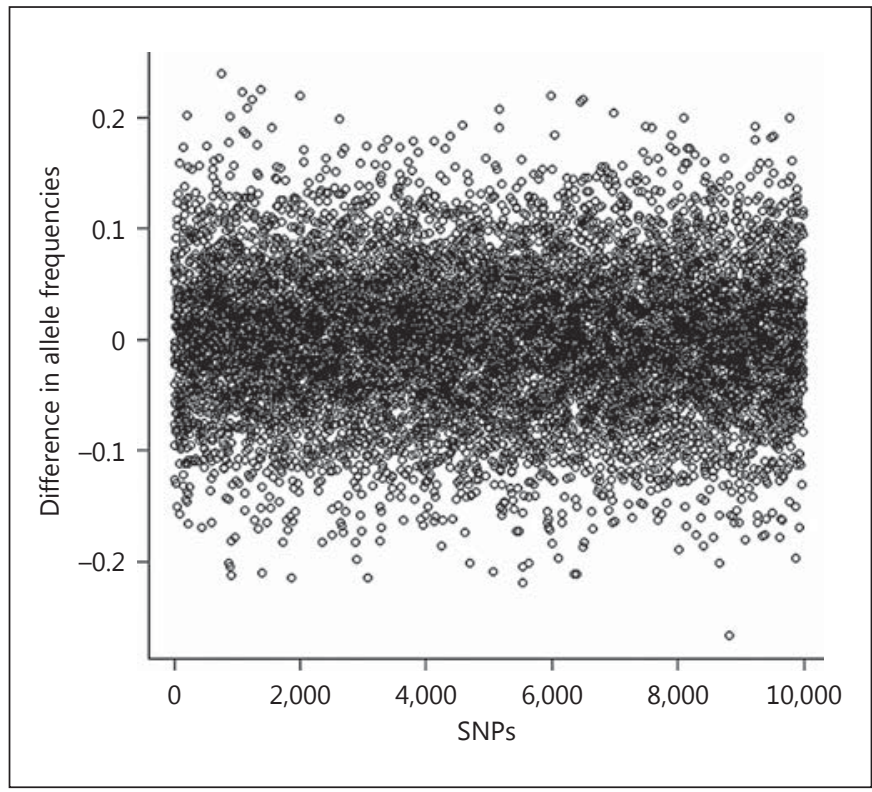

Fig. 1. Allele frequency differences between the 2 populations in 1 simulated data set.

Table 1. Empirical type I error of the original HWD trend test and the extended HWD trend test based on 10,000 replicates with a significance level of 0.05

\begin{tabular}{lll}
\hline $\begin{array}{l}\text { Allele frequency } \\
\text { differences }\end{array}$ & HWD trend test & $\begin{array}{l}\text { Extended HWD } \\
\text { trend test }\end{array}$ \\
\hline Random $^{\mathrm{a}}$ & 0.0502 & 0.0459 \\
$0.2-0.8^{\mathrm{b}}$ & 0.9809 & 0.0508 \\
\hline
\end{tabular}

a The allele frequency differences of the candidate null SNP between the 2 populations were randomly simulated based on $F_{s t}=$ 0.01 .

${ }^{\mathrm{b}}$ The allele frequency differences of the candidate null SNP between the 2 populations were set to 0.2 and 0.8 , respectively.

\section{FWER Evaluation}

To evaluate the FWERs of our proposed GSB procedures, we generated 100,000 replicates of the case-control data (with 10,000 plus 10 SNPs) under $H_{0}$ that no SNPs are associated with the disease (see Simulating Case-Control Data with Stratification above). The FWER was estimated as the proportion of replicates with at least 1 false discovery. We also estimated the FWERs of the extended HWD trend test and the GA trend test. For these individual SNP-based tests, the significance level for individual SNPs was set using Bonferroni correction, i.e. the 
Table 2. Empirical FWER based on 100,000 replicates with a nominal FWER of $0.01,0.05$ and 0.10

\begin{tabular}{|c|c|c|c|c|c|c|c|c|c|c|c|}
\hline FWER & $\begin{array}{l}\text { Extended } \\
\text { HWD } \\
\text { trend test }\end{array}$ & $\begin{array}{l}\text { GA trend } \\
\text { test }\end{array}$ & $\begin{array}{l}\text { GSB } \\
\text { procedure }\end{array}$ & \multicolumn{8}{|c|}{ Smooth-GSB procedure $(\lambda=)$} \\
\hline 0.05 & 0.0417 & 0.0478 & 0.0487 & 0.0478 & 0.0477 & 0.0476 & 0.0475 & 0.0473 & 0.0473 & 0.0473 & 0.0475 \\
\hline 0.10 & 0.0827 & 0.0927 & 0.0944 & 0.0942 & 0.0942 & 0.0939 & 0.0937 & 0.0937 & 0.0933 & 0.0931 & 0.0927 \\
\hline
\end{tabular}

For the individual SNP-based tests (extended HWD trend test and the GA trend test), the significance level for individual SNPs was set using Bonferroni correction, i.e. the nominal FWER was divided by the number of SNPs.

nominal FWER was divided by the number of SNPs. For the smooth-GSB procedure, different values for $\lambda$ from 0.55 to 0.9 were evaluated. The estimated FWERs are reported in table 2. We can see that all methods controlled the FWER well. We did not estimate the FWER of MAX3PC because of the high computational cost. The evaluation of the type I error of MAX3-PC can be found in So and Sham's paper [12].

\section{Power Evaluation}

To evaluate the power of the proposed methods, we simulated a causal SNP besides the 10,000 plus 10 noncausal SNPs as follows. At the causal SNP with allele $A$ and $B$, let $B$ denote the risk allele. We assume HardyWeinberg equilibrium in subpopulations 1 and 2. Suppose $p_{k}(k=1,2)$ is the frequency of allele $B$ in population $k$. The genotype frequencies of $(A A, A B, B B)$ for controls (population control) of population $k$ are set to $\left[\left(1-p_{k}\right)^{2}\right.$, $\left.2 p_{k}\left(1-p_{k}\right), p_{k}^{2}\right]$. Let the genetic relative risks be $R_{1}=$ $\operatorname{Pr}($ affected $\mid A B) / \operatorname{Pr}($ affected $\mid A A), R_{2}=\operatorname{Pr}($ affected $\mid B B) /$ $\operatorname{Pr}($ affected $\mid A A)$. When the genetic model is additive, multiplicative, dominant or recessive, we have $R_{2}=2$ $R_{1}-1, R_{2}=R_{1}^{2}, R_{1}=R_{2}$, or $R_{1}=1$, respectively. The genotype frequencies of $(A A, A B, B B)$ in cases is set to [ $(1-$ $\left.\left.p_{k}\right)^{2} / c, 2 p_{k}\left(1-p_{k}\right) R_{1} / c, p_{k}^{2} R_{2} / c\right]$, where $c=\left(1-p_{k}\right)^{2}+2 p_{k}$ $\left(1-p_{k}\right) R_{1}+p_{k}^{2} R_{2}$. With the specified genotype frequencies, cases and controls were sampled from the corresponding trinomial distribution with parameters [ $(1-$ $\left.\left.p_{k}\right)^{2} / c, 2 p_{k}\left(1-p_{k}\right) R_{1} / c, p_{k}^{2} R_{2} / c\right]$ and $\left[\left(1-p_{k}\right)^{2}, 2 p_{k}\left(1-p_{k}\right)\right.$, $p_{k}^{2}$ ], respectively. We compared the power of 5 different methods: (1) the extended HWD trend test, (2) the GA trend test as implemented in EIGENSTRAT, (3) the GSB procedure, (4) the smooth-GSB procedure, and (5) MAX3-PC. The top 10 PCs of the genome-wide genotypes were used as covariates in the GA trend test and MAX3-PC. We simulated data under the 4 genetic disease models: the additive, multiplicative, dominant and recessive models; and we considered different genotype relative risks. For each scenario, we simulated 10,000 replicates of the case-control data. The nominal FWER was set to be 0.05 . For the individual SNP-based tests (the extended HWD trend test, the GA trend test, and MAX3$\mathrm{PC}$ ), the corresponding significance level for each SNP was set to $0.05 / m$, where $m$ is the number of SNPs. The power was estimated as the proportion of replicates with identified significant SNPs.

To test the influence of different $\lambda$ values on the smooth-GSB procedure, we estimated the power of the smooth-GSB procedure with different $\lambda$ values ranging from 0.55 to 0.90 based on 10,000 replicated data sets. The results are reported in table 3 . We can see that the power of the smooth-GSB procedure is robust to different $\lambda$ values when $0.55 \leq \lambda \leq 0.9$. When $\lambda$ increased from 0.55 to 0.90 , the power of the smooth-GSB procedure slightly increased under the additive and the multiplicative models and slightly decreased under the dominant and recessive models. More specifically as $\lambda$ increased from 0.60 to 0.85 , the power increase of the smooth-GSB procedure under the additive and multiplicative models was usually less than 0.02 , and the power decrease under the dominant and recessive models was usually less than 0.03 . Given this, we would further suggest setting the $\lambda$ value in the smooth-GSB procedure such that $0.60 \leq \lambda \leq 0.85$. For example, we can set $\lambda=0.7$. Compared to the GA trend test, our smooth-GSB procedure has much higher power under both the recessive and dominant models. On the other hand, the smooth-GSB procedure (when $0.60 \leq$ $\lambda \leq 0.85$ ) has power very close to the GA trend test (the difference is often less than 0.02) under both the additive and multiplicative models. For example, under the recessive model with genotype relative risk $R_{2}=1.5$, the power of the smooth-GSB procedure with $\lambda=0.6$ was 0.531 , 
Table 3. Empirical power based on 10,000 replicates with a nominal FWER of 0.05

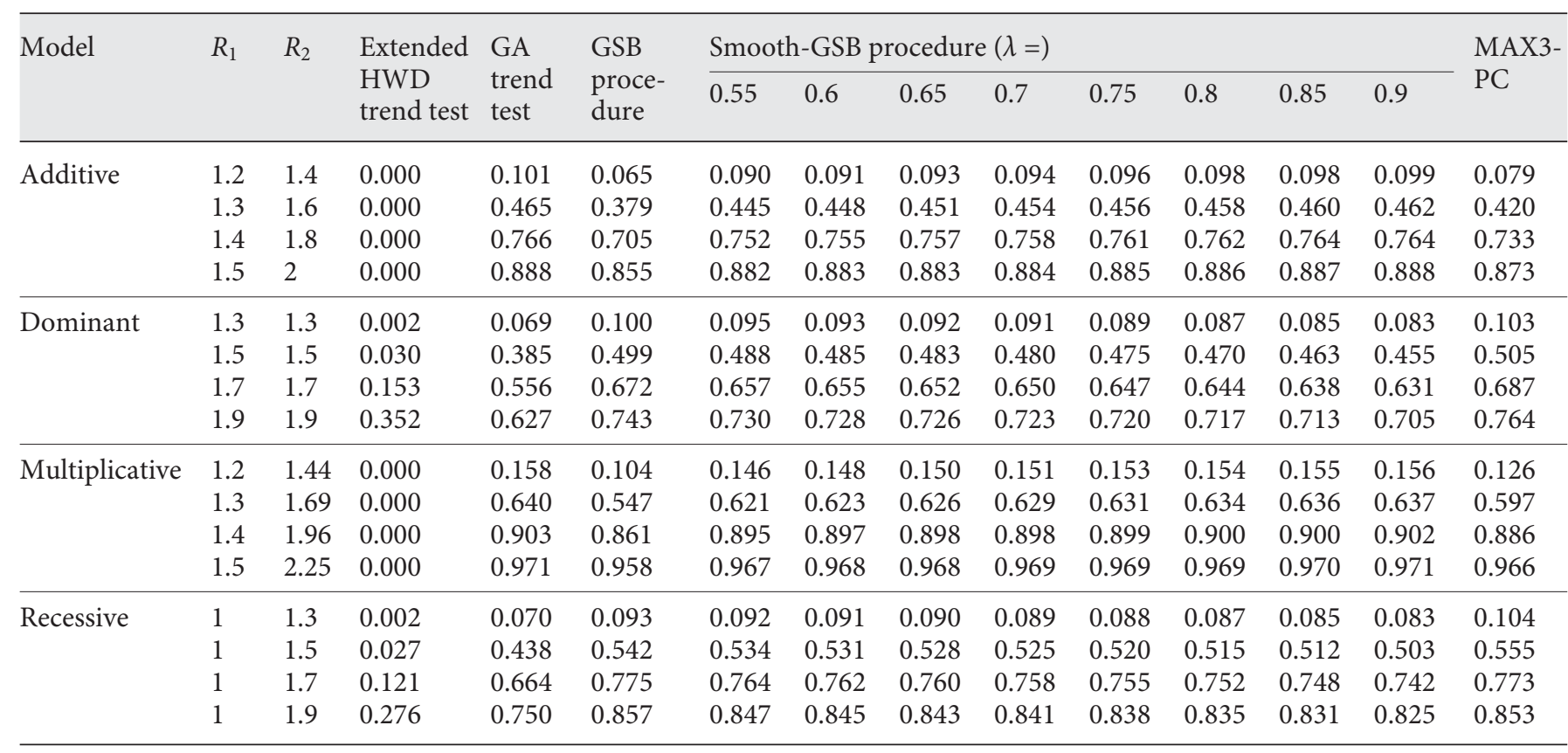

which was about $10 \%$ (in absolute terms) higher than the power of the GA trend test (0.438). We can also see that the robust test MAX3-PC by So and Sham [12] had relatively lower power than the GA trend test and our smoothGSB procedure under the additive and multiplicative models, while it had higher power than the other 2 methods under the dominant and recessive models. For example, under the additive model with genotype relative risk $R_{1}=1.3$, the power of the GA trend test, the smoothGSB procedure (with $\lambda=0.6$ ) and MAX-PC were 0.465 , 0.448 , and 0.420 , respectively. The power of MAX3-PC was $4.5 \%$ (in absolute terms) lower than that of the GA trend test.

Another advantage of the smooth-GSB procedure is that it is much faster than MAX3-PC. On a computer with a 3.47-GHz CPU, with the PCs already computed, our R implementation of the smooth-GSB procedure for GWAS took less than $15 \mathrm{~s}$ to process 10,000 plus 10 SNPs, while MAX3-PC implemented in the robust SNP package [12] took about $1,100 \mathrm{~s}$, if the absolute error of $\mathrm{p}$ values was set to $10^{-6}$. It took about $2,500 \mathrm{~s}$, if the absolute error of $\mathrm{p}$ values was set to $10^{-8}$. This means that for GWAS with about $1,000,000$ SNPs, MAX3-PC takes about $70 \mathrm{~h}$ with a $\mathrm{p}$ value accuracy level of $10^{-8}$, while the smooth-GSB procedure takes less than $25 \mathrm{~min}$.

\section{Application to WTCCC Data}

The WTCCC data contain 7 different data sets of major diseases [13]. To evaluate the performance of our proposed method on real data, we chose 4 data sets from the WTCCC data: on bipolar disorder (BD), coronary artery disease (CAD), Crohn's disease (CD), and type 2 diabetes (T2D). Each of the 4 data sets has a reported genomic control inflation factor $>1.06$ [13]; therefore, there may be population stratification in each of these data sets. There are about 3,000 controls and about 2,000 cases in each data set.

We did a quality control according to the description in the WTCCC paper [13] to filter out some individuals and SNPs. The total number of SNPs after the quality control was about 400,000. Following the suggestion of the WTCCC paper [13], we used only 2 top PCs as covariates to correct for population stratification in the GA trend test and MAX3-PC. These PCs were calculated based on about 190,000 SNPs, which were obtained by pruning the whole genome SNPs using the software PLINK [24]. For each of the 3 single marker-based tests (the extended HWD trend test, the GA trend test and MAX3-PC), we can calculate a $p$ value at each of the about 400,000 SNPs. But for the smooth-GSB procedure, we can only calculate an adjusted p value for each SNP (see GSB 
Table 4. $p$ values for the most significant SNPs in the regions identified by the GA trend test, the smooth-GSB procedure or MAX3-PC in 4 WTCCC data sets with population stratification

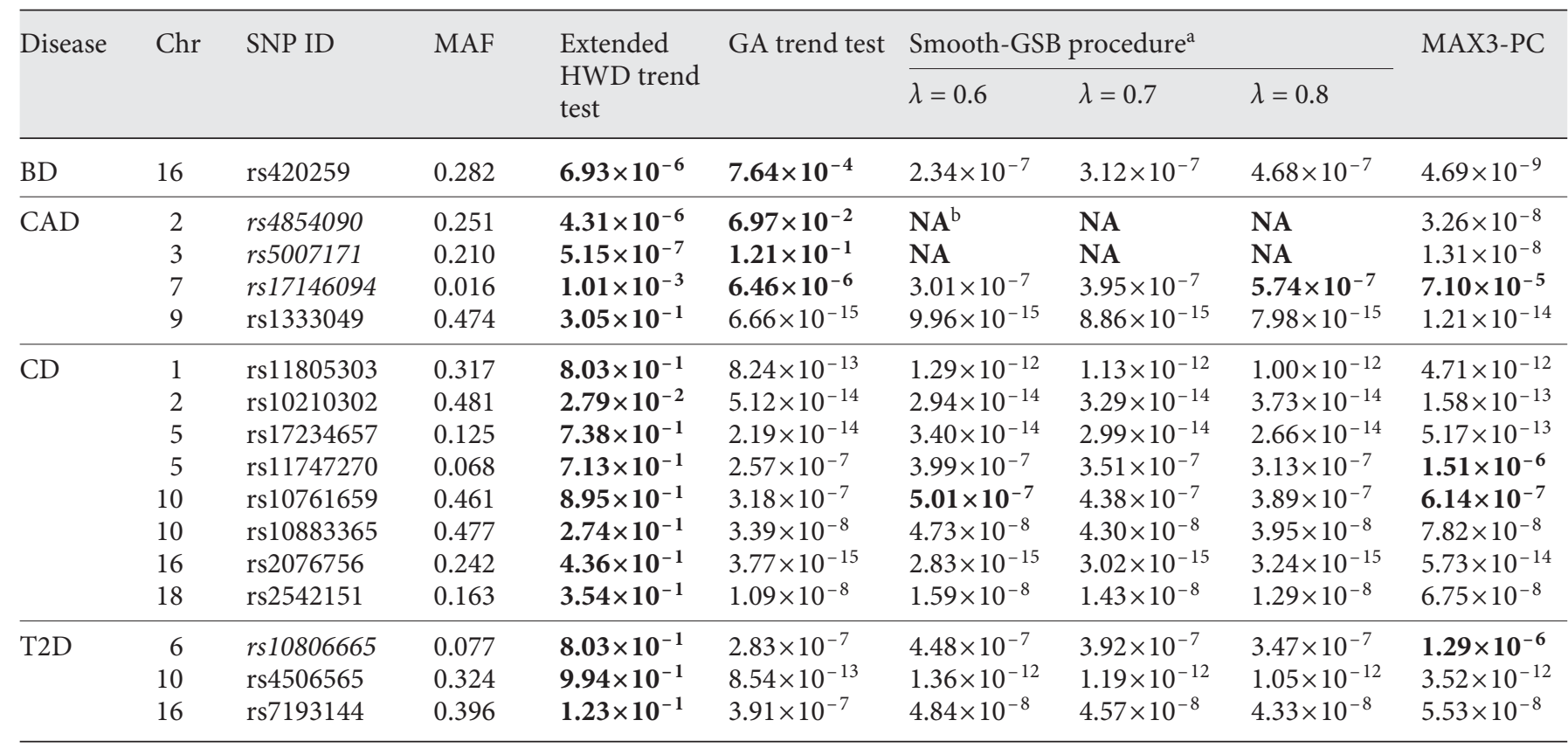

$\mathrm{Chr}=$ Chromosome; $\mathrm{MAF}=$ minor allele frequency.

Only those SNPs with the smallest $\mathrm{p}$ value in each significant region are listed.

SNP IDs in italics indicate that the corresponding region is not listed in table 3 of the WTCCC paper [13], which lists the regions of the strongest association signals. $\mathrm{p}$ values in bold did not reach the genome-wide significance level of $5 \times 10^{-7}$.

${ }^{a} \mathrm{p}$ values for the smooth-GSB procedure were calculated as the adjusted $\mathrm{p}$ values divided by the number of tests.

${ }^{\mathrm{b}} \mathrm{NA}=$ Not applicable. The adjusted $\mathrm{p}$ value is equal to 1 and therefore the $\mathrm{p}$ value for the individual SNP was not calculated.
Procedure for Multiple Testing above). In order to compare the adjusted $\mathrm{p}$ value with the $\mathrm{p}$ values from the 3 single marker-based tests, we define a $p$ value for each SNP in the smooth-GSB procedure as the adjusted $\mathrm{p}$ value divided by the number of tests $(m)$; however, if the adjusted $p$ value at a SNP is 1 , then we will not define a $p$ value for that SNP because $H_{0}$ will not be rejected at that SNP. When assuming the independence of markers, for the smooth-GSB procedure, comparing the adjusted $\mathrm{p}$ value with the nominal FWER $\alpha$ is equivalent to comparing the new defined $\mathrm{p}$ value with $\alpha / m$.

Following the suggestion of the WTCCC paper [13], we used a threshold of $5 \times 10^{-7}$ for individual SNPs. Table 4 lists the significant SNPs identified by at least 1 of the 3 methods: the GA trend test, the smooth-GSB procedure, and MAX3-PC. For each significant region, only those SNPs with the smallest $p$ value are listed. We can see that all SNPs detected by the GA trend test were also detected by the smooth-GSB procedure with different $\lambda$ values: 0.6 , 0.7 , and 0.8 ; the only exception is SNP rs10761659 that was not identified by the smooth-GSB procedure with $\lambda=$ 0.6 ( $\mathrm{p}$ value $=5.01 \times 10^{-7}$, which is very close to the threshold of $5 \times 10^{-7}$ ). For BD, SNP rs420259 was detected by both the smooth-GSB procedure and MAX3-PC, but not by the GA trend test. This is reasonable because the bestfitting genetic model for SNP rs420259 is recessive [13]. As we described earlier, the GA trend test has low power under the recessive model. In addition, 2 SNPs, rs 11747270 (for CD) and rs 10806665 (for T2D), and their corresponding regions were detected by both the GA trend test and the smooth-GSB procedure, but were not detected by MAX3-PC. The reason for this might be that the underlying disease models at these 2 SNPs are additive and MAX3PC has relatively lower power than the other 2 methods under the additive model. We note that SNP rs10806665 (for T2D) was not identified as significant by the WTCCC paper [13]. We also note that for CAD, 2 SNPs, rs 4854090 and rs5007171, were detected by MAX3-PC but not by the GA trend test and smooth-GSB procedure. The underlying disease models at these 2 SNPs might be recessive or 
dominant, because under these 2 models, MAX3-PC has higher power than the other 2 methods. These 2 SNPs and their corresponding regions were not reported as significant by the WTCCC paper [13].

\section{Discussion}

In this study, we proposed a fast and robust association test procedure (the smooth-GSB procedure), which can be applied to GWAS data with case-control designs in the presence of subpopulations. As showed in the simulation studies, the smooth-GSB procedure can have significantly higher power than the GA trend test implemented in EIGENSTRAT when the underlying genetic model is dominant or recessive. It can also have power comparable to that of the GA trend test when the underlying genetic model is additive or multiplicative. Our simulation studies indicate that the existing robust test, MAX3-PC, can be computationally intensive for GWAS with a large number of SNPs and can have relatively lower power than the GA trend test when the underlying disease model is additive or multiplicative. Our proposed method can be viewed as an extension of our previously proposed GSB procedure for GWAS in homogeneous populations to account for population stratification [25].

The proposed smooth-GSB procedure is not appropriate for the analysis of rare variants (e.g. with a minor allele frequency $<0.05)$. When the minor allele frequency is $<0.05$, the extended HWD trend test may not produce accurate $\mathrm{p}$ values.

In our proposed smooth-GSB procedure, the parameter $\lambda(0 \leq \lambda \leq 1)$ should be set empirically. If we choose $\lambda=1$, then the smooth-GSB procedure becomes the sequential rejective procedure by Holm [20] using the GA trend test and is approximately equivalent to the GA trend test with Bonferroni correction (if the number of markers is large enough such as in a GWAS analysis). When $\lambda=1$, the smooth-GSB procedure has the highest power if the underlying disease model is additive or multiplicative, but has relatively low power if the underlying disease model is recessive or dominant. On the other hand, if we choose $\lambda=0$, the smooth-GSB procedure becomes the GSB procedure (see GSB Procedure for GWAS in Case-Control Data with Population Stratification above), which has the highest power when the disease model is recessive or dominant, but has lower power than the GA trend test if the underlying disease model is additive or multiplicative. In real data analysis, usually we do not know the true disease models. Since most significant
SNPs reported in the literature so far have demonstrated additive effects, based on our simulation studies, we would suggest choosing the $\lambda$ value such that $0.6 \leq \lambda \leq$ 0.85 . For example, we can set $\lambda=0.7$. As indicated by the results of our simulation studies, when the $\lambda$ values change from 0.6 to 0.85 , the power of our smooth-GSB procedure is: (a) very robust to different $\lambda$ values under different disease models; (b) very close to that of the GA trend test if the underlying disease model is additive or multiplicative, and (c) much higher than that of the GA trend test if the underlying disease model is recessive or dominant.

We note that in the extended HWD trend test, any proper clustering algorithm to partition individuals into subpopulations can be applied, such as the more computationally intensive method proposed by Pritchard et al. [26]. The $k$-means clustering method used in this paper has the advantage of low computation cost. In the extended HWD trend test, we applied the $k$-means clustering algorithm to the top PCs of the genotype data to partition the individuals in the case-control data into several clusters corresponding to homogeneous subpopulations. This $k$-means clustering may not be appropriate for data from admixed populations, for which different partitioning methods may be needed. For example, we can partition individuals by the number of alleles at a marker inherited from an ancestral population. This partitioning method was used by Shriner et al. [27] in association studies using admixed populations.

In this paper, we assume independence of markers in GWAS by ignoring the dependence among markers. The proposed methods under this assumption can be relatively conservative when applied to GWAS data analysis due to ignoring the $\mathrm{LD}$ among markers. Accounting for $\mathrm{LD}$ among dense markers may increase the power of association study methods in detecting causal variants. In our future study, we plan to incorporate SNP set- (or gene-) based association tests, e.g. SKAT [28], which can account for dependence among markers, into our smoothGSB procedure.

\section{Appendix}

Evaluation of FWER of the GSB Procedures by Holm [20] for Multiple Testing with Weights Weakly Correlated with the Tests

Here we showed by simulation studies that in some situations, even though the weights are weakly correlated with the $m$ tests, the GSB procedure by Holm [20] for $m$ independent hypotheses, $H_{1}$, $H_{2}, \ldots, H_{m}$, can still control FWER well (see also GSB Procedure for Multiple Testing above). Assuming all null hypotheses, $H_{1}, H_{2}$, 
Table 5. Empirical FWER of the GSB procedure by Holm [20] for 1,000 tests with weights weakly correlated with the tests (based on $10^{7}$ replicated data sets)

\begin{tabular}{llll}
\hline$\rho_{\mathrm{s}}$ & \multicolumn{3}{l}{ Nominal FWER $\alpha^{\mathrm{a}}$} \\
\cline { 2 - 4 } & 0.01 & 0.05 & 0.1 \\
\hline 0.005 & 0.0103 & 0.0505 & 0.0987 \\
0.01 & 0.0101 & 0.0495 & 0.0965 \\
\hline
\end{tabular}

$\rho_{s}=$ Spearman's rank correlation coefficient between the test statistics, respectively.

a The nominal FWER used to reject a null hypothesis. between the pair of $q$ and $p$ values $\left(q_{j}\right.$ and $\left.p_{j}\right)$. Once the pair of $q$ and $p$ values was simulated for each test, we had 1,000 pairs of $q$ and $p$ values corresponding to 1,000 tests. We calculated weights by using the $q$ values, and then we applied the GSB procedure to the 1,000 tests. We replicated this simulation procedure $10^{7}$ times and therefore generated $10^{7}$ replicated 'data sets', each consisting of 1,000 pairs of $q$ and $p$ values. We estimated the FWER of the GSB procedure over these $10^{7}$ replicated data sets with the nominal FWER of $0.01,0.05$ and 0.1 . The FWER was estimated as the proportion of replicates with at least 1 false discovery. The results are showed in table 5. From the results we can see that the FWER of the GSB procedure was well controlled when Spearman's rank correlation coefficient $\rho_{\mathrm{s}}$ is $\leq 0.01$ (i.e. the $q$ and $p$ values were weakly correlated).

\section{Acknowledgements}

This research was supported by the National Institutes of Health grants: R01GM073766 from the National Institute of General Medical Sciences, U01HL101064 from the National Heart, Lung, and Blood Institute, and R01LM011177 from the National Library of Medicine. The research was also partly supported by the National Center for Advancing Translational Sciences (grant UL1TR000058). This study makes use of data generated by the Wellcome Trust Case-Control Consortium. A full list of the investigators who contributed to the generation of the data is available from www.wtccc.org.uk. Funding for the project was provided by the Wellcome Trust under award 076113 and 085475 .

\section{References}

1 Reich DE, Goldstein DB: Detecting association in a case-control study while correcting for population stratification. Genet Epidemiol 2001;20:4-16.

$\checkmark 2$ Price AL, Zaitlen NA, Reich D, Patterson N: New approaches to population stratification in genome-wide association studies. Nat Rev Genet 2010;11:459-463.

-3 Price AL, Patterson NJ, Plenge RM, Weinblatt ME, Shadick NA, Reich D: Principal components analysis corrects for stratification in genome-wide association studies. Nat Genet 2006;38:904-909.

-4 Campbell CD, Ogburn EL, Lunetta KL, Lyon HN, Freedman ML, Groop LC, Altshuler D, Ardlie KG, Hirschhorn JN: Demonstrating stratification in a European American population. Nat Genet 2005;37:868-872.

5 Devlin B, Roeder K: Genomic control for association studies. Biometrics 1999;55:9971004.

6 Pritchard JK, Stephens M, Rosenberg NA, Donnelly P: Association mapping in structured populations. Am J Hum Genet 2000;67: 170-181.
7 Freidlin B, Zheng G, Li Z, Gastwirth JL: Trend tests for case-control studies of genetic markers: power, sample size and robustness. Hum Hered 2002;53:146-152.

8 González JR, Carrasco JL, Dudbridge F, Armengol L, Estivill X, Moreno V: Maximizing association statistics over genetic models. Genet Epidemiol 2008;32:246-254.

$>$ Wang K, Sheffield VC: A constrained-likelihood approach to marker-trait association studies. Am J Hum Genet 2005;77:768-780.

10 Joo J, Kwak M, Zheng G: Improving power for testing genetic association in case-control studies by reducing the alternative space. Biometrics 2010;66:266-276.

11 Armitage P: Tests for linear trends in proportions and frequencies. Biometrics 1955;11: 375-386.

12 So HC, Sham PC: Robust association tests under different genetic models, allowing for binary or quantitative traits and covariates. Behav Genet 2011;41:768-775.
13 The Wellcome Trust Case Control Consortium: Genome-wide association study of 14,000 cases of seven common diseases and 3,000 shared controls. Nature 2007;447:661678 .

14 Song K, Elston RC: A powerful method of combining measures of association and Hardy-Weinberg disequilibrium for fine-mapping in case-control studies. Stat Med 2006; 25:105-126.

15 MacQueen J: Some methods for classification and analysis of multivariate observations; in Cam LM Le, Neyman J (eds): Proceedings of the Fifth Berkeley Symposium on Mathematical Statistics and Probability. Berkeley, University of California Press, 1967, pp 281-297.

16 Hennig C: fpc: flexible procedures for clustering. R package version 2.0-3. http://CRAN.Rproject.org/package $=\mathrm{fpc}, 2010$.

17 Duda RO, Hart PE: Pattern Classification and Scene Analysis, ed 1. New York, John Wiley \& Sons, 1973.

18 Calinski RB, Harabasz J: A dendrite method for cluster analysis. Comm Stat 1974;3:1-27. 
19 Mägi R, Morris AP: GWAMA: software for genome-wide association meta-analysis. BMC Bioinformatics 2010;11:288.

20 Holm S: A simple sequentially rejective multiple test procedure. Scand J Stat 1979;6:6570.

21 Westfall PH, Yong SS: Resampling-Based Multiple Testing: Examples and Methods for p-Value Adjustment. New York, John Wiley \& Sons, 1993.

22 Roeder K, Devlin B, Wasserman L: Improving power in genome-wide association studies: weights tip the scale. Genet Epidemiol 2007; 31:741-747.
23 Joo J, Kwak M, Ahn K, Zheng G: A robust genome-wide scan statistic of the Wellcome Trust Case-Control Consortium. Biometrics 2009;65:1115-1122.

24 Purcell S, Neale B, Todd-Brown K, Thomas L, Ferreira MA, Bender D, Maller J, Sklar P, de Bakker PI, Daly MJ, Sham PC: PLINK: a tool set for whole-genome association and population-based linkage analyses. Am J Hum Genet 2007;81:559-575.

25 Gao G, Kang G, Wang J, Chen W, Qin H, Jiang B, Li Q, Sun C, Liu N, Archer KJ, Allison DB: A generalized sequential Bonferroni procedure using smoothed weights for genomewide association studies incorporating information on Hardy-Weinberg disequilibrium among cases. Hum Hered 2011;73:1-13.
26 Pritchard JK, Stephens M, Donnelly P: Inference of population structure using multilocus genotype data. Genetics 2000;155:945-959.

27 Shriner D, Adeyemo A, Rotimi CN: Joint ancestry and association testing in admixed individuals. PLoS Comput Biol 2011;7: e10002325.

28 Wu MC, Lee S, Cai T, Li Y, Boehnke M, Lin $\mathrm{X}$ : Rare variant association testing for sequencing data with the sequence kernel association test (SKAT). Am J Hum Genet 2011; 89:82-93.

29 Nelsen RB: An Introduction to Copulas, ed 2. New York, Springer, 2006. 